\title{
Influence of nucleonic motion in Relativistic Fermi Gas inclusive responses
}

\author{
L. Alvarez-Ruso ${ }^{1}$, M.B. Barbaro ${ }^{1}$, T.W. Donnelly ${ }^{2}$ and A. Molinari ${ }^{1}$ \\ ${ }^{1}$ Dipartimento di Fisica Teorica, Università di Torino and INFN, Sezione di Torino \\ Via P. Giuria 1, 10125 Torino, ITALY \\ ${ }^{2}$ Center for Theoretical Physics, \\ Laboratory for Nuclear Science and Department of Physics \\ Massachusetts Institute of Technology, Cambridge, MA 02139, USA
}

\begin{abstract}
Impulsive hadronic descriptions of electroweak processes in nuclei involve two distinctly different elements: one stems from the nuclear many-body physics - the medium — which is rather similar for the various inclusive response functions, and the other embodies the responses of the hadrons themselves to the electroweak probe and varies with the channel selected. In this letter we investigate within the context of the relativistic Fermi gas in both the quasi-elastic and $N \rightarrow \Delta$ regimes the interplay between these two elements. Specifically, we focus on expansions in the one small parameter in the problem, namely, the momentum of a nucleon in the initial wave function compared with the hadronic scale, the nucleon mass. Both parity-conserving and -violating inclusive responses are studied and the interplay between longitudinal $(L)$ and transverse ( $T$ and $\left.T^{\prime}\right)$ contributions is highlighted.
\end{abstract}

PACS: 25.30.Rw, 14.20.Gk, 24.10.Jv, 24.30.Gd, 13.40.Gp

Keywords: Nuclear reactions; Inclusive electron scattering; Relativistic Fermi Gas. 
In this letter we explore the influence of relativistic kinematics and of the medium on inclusive electron-nucleus scattering in the context of the Relativistic Fermi Gas (RFG). In spite of its simplicity the RFG is covariant, a requirement that is clearly relevant for analyses of modern medium- to high-energy nuclear electron scattering experiments. In past work compact, transparent expressions have been obtained for the longitudinal $(L)$ and transverse $(T)$ responses in parity-conserving $(\mathrm{PC})$ scattering and the $L, T$ and axial $\left(T^{\prime}\right)$ response functions in parity-violating (PV) scattering. Both the quasi-elastic (QE) and $\Delta$ regions have been studied [1, 2, 3]. In all cases the responses factorize as follows

$$
\begin{aligned}
R^{L, T}(q, \omega) & =R_{0}(q, \omega) U^{L, T}(q, \omega) \\
\tilde{R}^{L, T, T^{\prime}}(q, \omega) & =R_{0}(q, \omega) \tilde{U}^{L, T, T^{\prime}}(q, \omega)
\end{aligned}
$$

for the PC and PV sectors, respectively, providing a unified approach for treatments of electroweak interactions at high inelasticity. In (11) and (2) the $\Delta$ is viewed as a stable particle, but later we shall model its decay by including its width.

Defining an overall constant $C \equiv 3 \mathcal{N} /\left(4 \kappa m_{N} \eta_{F}^{3}\right)$, here we have a universal factor (the same for all the responses we consider)

$$
R_{0}(q, \omega)=C \xi_{F}\left(1-\psi^{2}\right),
$$

which scales in the region where the RFG is expected to be a reasonable model, specifically at $q>2 p_{F}$ where Pauli blocking effects are absent [4, 5, 6]. That is, it depends only upon a single variable $\psi$, whose square is given by

$$
\psi^{2}=\frac{\epsilon_{0}-1}{\xi_{F}}=\frac{1}{\xi_{F}}\left(\kappa \sqrt{\frac{1}{\tau}+\rho^{2}}-\lambda \rho-1\right),
$$

linked to the minimum energy $\epsilon_{0}$ a nucleon can have in the RFG to participate in the response of the system to an external electroweak field. In contrast to $R_{0}$, the functions $U^{L, T, T^{\prime}}$ depend upon the specific electron-nucleon process of interest.

In the above $\mathcal{N}$ is the number of nucleons t, $\eta_{F}=\sqrt{\xi_{F}\left(\xi_{F}+2\right)}=p_{F} / m_{N}$ is the dimensionless Fermi momentum and the dimensionless four-momenta

$$
\eta^{\mu}=(\epsilon, \vec{\eta})=\left(\sqrt{1+p^{2} / m_{N}^{2}}, \frac{\vec{p}}{m_{N}}\right), \quad \kappa^{\mu}=(\lambda, \vec{\kappa})=\left(\frac{\omega}{2 m_{N}}, \frac{\vec{q}}{2 m_{N}}\right)
$$

have been introduced for the initial nucleon and the exchanged boson, respectively, with $\tau=\kappa^{2}-\lambda^{2}$. Moreover, $\rho$ is an inelasticity parameter given by [2]

$$
\rho=1+\frac{1}{4 \tau}\left(\mu^{2}-1\right)
$$

with $\mu \equiv m_{\Delta} / m_{N}$, which reduces to unity in the nucleonic quasi-elastic sector.

\footnotetext{
${ }^{1}$ Actually in the nucleonic sector one should add, with the appropriate form factors, the responses with $\mathcal{N}=Z$ and $\mathcal{N}=N$ separately; in the $\Delta$ sector one should simply set $\mathcal{N}=A$.
} 
The response functions (11) and (2) are obtained as specific components of the nuclear tensor

$$
W_{\mu \nu}=\frac{3 \mathcal{N}}{4 \pi m_{N} \eta_{F}^{3}} \int \frac{d \vec{\eta}}{\epsilon \epsilon^{\prime}} \delta\left(2 \lambda-\epsilon^{\prime}+\epsilon\right) \theta\left(\eta_{F}-|\vec{\eta}|\right) f_{\mu \nu}(\vec{\eta}),
$$

which incorporates the single-nucleon responses, expressed via the single-nucleon tensor $f_{\mu \nu}$, over the allowed energy range. In particular, one has

$$
\begin{aligned}
R^{L} & =W_{00}=C \int_{\epsilon_{0}}^{\epsilon_{F}} f_{00}\left(\epsilon, \theta_{0}\right) d \epsilon \\
R^{T} & =W_{11}+W_{22}=C \int_{\epsilon_{0}}^{\epsilon_{F}}\left[f_{11}\left(\epsilon, \theta_{0}\right)+f_{22}\left(\epsilon, \theta_{0}\right)\right] d \epsilon \\
\tilde{R}^{L} & =\tilde{W}_{00}=C \int_{\epsilon_{0}}^{\epsilon_{F}} \tilde{f}_{00}\left(\epsilon, \theta_{0}\right) d \epsilon \\
\tilde{R}^{T} & =\tilde{W}_{11}+\tilde{W}_{22}=C \int_{\epsilon_{0}}^{\epsilon_{F}}\left[\tilde{f}_{11}\left(\epsilon, \theta_{0}\right)+\tilde{f}_{22}\left(\epsilon, \theta_{0}\right)\right] d \epsilon \\
\tilde{R}^{T^{\prime}} & =-i \tilde{W}_{12}=-i C \int_{\epsilon_{0}}^{\epsilon_{F}} \tilde{f}_{12}\left(\epsilon, \theta_{0}\right) d \epsilon,
\end{aligned}
$$

where

$$
\cos \theta_{0}=\frac{\lambda \epsilon-\tau \rho}{\kappa \eta}
$$

and

$$
f_{\mu \nu}\left(\epsilon, \theta_{0}\right)=-w_{1}(\tau)\left(g_{\mu \nu}+\frac{\kappa_{\mu} \kappa_{\nu}}{\tau}\right)+w_{2}(\tau) V_{\mu} V_{\nu}-2 i w_{3}(\tau) \epsilon_{\mu \nu \rho \sigma} \kappa^{\rho} V^{\sigma},
$$

with $V_{\mu}=\eta_{\mu}+\kappa_{\mu} \rho$. Note that (13) follows from energy conservation and the lower limit of integration is defined by (4). The single-nucleon tensor (14) is valid for both the quasielastic process and the $N \rightarrow \Delta$ transition, but of course the $w_{i}$ 's are different in the two cases. In the QE region these are [四]

$$
\begin{aligned}
w_{1, a}(\tau) & =\tau G_{M, a}^{2}(\tau) \\
w_{2, a}(\tau) & =\frac{1}{1+\tau}\left[G_{E, a}^{2}(\tau)+\tau G_{M, a}^{2}(\tau)\right]
\end{aligned}
$$

in the PC sector and

$$
\begin{aligned}
\tilde{w}_{1, a}(\tau) & =\tau G_{M, a}(\tau) \tilde{G}_{M, a}(\tau) \\
\tilde{w}_{2, a}(\tau) & =\frac{1}{1+\tau}\left[G_{E, a}(\tau) \tilde{G}_{E, a}(\tau)+\tau G_{M, a}(\tau) \tilde{G}_{M, a}(\tau)\right] \\
\tilde{w}_{3, a}(\tau) & =G_{M, a}(\tau) \tilde{G}_{A, a}(\tau)
\end{aligned}
$$

in the PV one, the index $a$ referring to protons or neutrons.

For the $\Delta$ sector one has instead

$$
\begin{aligned}
w_{1, \Delta}(\tau) & =\frac{1}{16}\left[4 \tau+(\mu-1)^{2}\right](\mu+1)^{2}\left[G_{M, \Delta}^{2}(\tau)+3 G_{E, \Delta}^{2}(\tau)\right] \\
w_{2, \Delta}(\tau) & =\frac{1}{16} \frac{4 \tau+(\mu-1)^{2}}{1+\tau \rho^{2}}(\mu+1)^{2}\left[G_{M, \Delta}^{2}(\tau)+3 G_{E, \Delta}^{2}(\tau)+\frac{4 \tau}{\mu^{2}} G_{C, \Delta}^{2}(\tau)\right]
\end{aligned}
$$


in the PC sector [2] and

$$
\begin{aligned}
\tilde{w}_{1, \Delta}(\tau)= & \frac{1}{16}\left[4 \tau+(\mu-1)^{2}\right](\mu+1)^{2} \\
& \times\left[G_{M, \Delta}(\tau) \tilde{G}_{M, \Delta}(\tau)+3 G_{E, \Delta}(\tau) \tilde{G}_{E, \Delta}(\tau)\right] \\
\tilde{w}_{2, \Delta}(\tau)= & \frac{1}{16} \frac{4 \tau+(\mu-1)^{2}}{1+\tau \rho^{2}}(\mu+1)^{2} \\
& \times\left[G_{M, \Delta}(\tau) \tilde{G}_{M, \Delta}(\tau)+3 G_{E, \Delta}(\tau) \tilde{G}_{E, \Delta}(\tau)+\frac{4 \tau}{\mu^{2}} G_{C, \Delta}(\tau) \tilde{G}_{C, \Delta}(\tau)\right] \\
\tilde{w}_{3, \Delta}(\tau)= & \frac{1}{4}\left(\mu^{2}-1\right)\left[3 G_{E, \Delta}(\tau) \tilde{G}_{M, \Delta}^{A}(\tau)+G_{M, \Delta}(\tau) \tilde{G}_{E, \Delta}^{A}(\tau)\right]
\end{aligned}
$$

in the PV one [3]. The parametrizations of the elastic form factors are given in [1], while those for the $N \rightarrow \Delta$ transitions are the following:

$$
\begin{aligned}
G_{E, \Delta}(\tau) & =-0.03 f(\tau) \\
G_{M, \Delta}(\tau) & =2.97 f(\tau) \\
G_{C, \Delta}(\tau) & =0 \\
\tilde{G}_{E, \Delta}(\tau) & =\beta_{V}^{T=1} G_{E, \Delta}(\tau) \\
\tilde{G}_{M, \Delta}(\tau) & =\beta_{V}^{T=1} G_{M, \Delta}(\tau) \\
\tilde{G}_{C, \Delta}(\tau) & =\beta_{V}^{T=1} G_{C, \Delta}(\tau) \\
\tilde{G}_{E, \Delta}^{A}(\tau) & =2.22 G_{D}^{A}(\tau) \\
\tilde{G}_{M, \Delta}^{A}(\tau) & =0
\end{aligned}
$$

where $f(\tau)=G_{E, p}(\tau)\left(1+\lambda_{\Delta} \tau\right)^{-1 / 2}$, with $\lambda_{\Delta}=1.0$ and $\beta_{V}^{T=1}=1-2 \sin ^{2} \theta_{W}=0.55$.

The energy integrations in $(\$ 12)$ are easily performed and yield the well-known results [1, [7, 3]

$$
\begin{aligned}
& U^{L}(\kappa, \tau)=\frac{\kappa^{2}}{\tau}\left[\left(1+\tau \rho^{2}\right) w_{2}(\tau)-w_{1}(\tau)+w_{2}(\tau) \mathcal{D}^{L}(\kappa, \tau)\right] \\
& U^{T}(\kappa, \tau)=2 w_{1}(\tau)+w_{2}(\tau) \mathcal{D}^{T}(\kappa, \tau) \\
& \tilde{U}^{L}(\kappa, \tau)=\frac{\kappa^{2}}{\tau}\left[\left(1+\tau \rho^{2}\right) \tilde{w}_{2}(\tau)-\tilde{w}_{1}(\tau)+\tilde{w}_{2}(\tau) \mathcal{D}^{L}(\kappa, \tau)\right] \\
& \tilde{U}^{T}(\kappa, \tau)=2 \tilde{w}_{1}(\tau)+\tilde{w}_{2}(\tau) \mathcal{D}^{T}(\kappa, \tau) \\
& \tilde{U}^{T^{\prime}}(\kappa, \tau)=2 \sqrt{\tau\left(\tau \rho^{2}+1\right)} \tilde{w}_{3}(\tau)\left[1+\mathcal{D}^{T^{\prime}}(\kappa, \tau)\right]
\end{aligned}
$$

where the functions $\mathcal{D}^{L, T, T^{\prime}}$ are such as to vanish in the limit $\xi_{F} \sim \eta_{F}^{2} \rightarrow 0$. Note that expressions such as (33) contain factors of the type $\kappa^{2} / \tau$. If the $\kappa^{2}$ in these factors were also expanded using, for example, $\tau, \eta$ and $\rho$ as independent variables, then terms linear in $\eta$ (and hence $\eta_{F}$ ) would occur. However, when written in the form given here using both 
$\kappa$ and $\tau$, more compact expressions result and the corrections - the terms containing the $\mathcal{D}$ 's - incur only small contributions of order $\eta_{F}^{2}$, namely, only quadratic in the one small parameter at our disposal.

We now explore the impact of the nucleonic motion on the inclusive responses. We begin by expanding the tensor $f_{\mu \nu}$ in the dimensionless nucleon momentum $\eta$ up to linear order, in the same spirit as in [8, 7, 2] where expansions have been performed for the spinor matrix elements of the nucleon electromagnetic single-nucleon and meson-exchange currents. Importantly, in the framework of this expansion one has that

$$
\kappa \eta \cos \theta_{0} \simeq \lambda-\tau \rho
$$

if terms higher than linear order are neglected. Since the $w_{i}$ 's only depend upon the external variable $\tau$, and the leading contribution in the nucleonic momentum expansion is quadratic for $\mathcal{D}^{L, T, T^{\prime}}$, as we shall see, then the following expressions for the $U$ functions are obtained from (33)-(37)

$$
\begin{aligned}
& U^{L}(\kappa, \tau) \simeq\left[\left(1+\tau \rho^{2}\right)+2 \rho(\lambda-\tau \rho)\right]\left[\left(1+\tau \rho^{2}\right) w_{2}(\tau)-w_{1}(\tau)\right] \\
& U^{T}(\kappa, \tau) \simeq 2 w_{1}(\tau) \\
& \tilde{U}^{L}(\kappa, \tau) \simeq\left[\left(1+\tau \rho^{2}\right)+2 \rho(\lambda-\tau \rho)\right]\left[\left(1+\tau \rho^{2}\right) \tilde{w}_{2}(\tau)-\tilde{w}_{1}(\tau)\right] \\
& \tilde{U}^{T}(\kappa, \tau) \simeq 2 \tilde{w}_{1}(\tau) \\
& \tilde{U}^{T^{\prime}}(\kappa, \tau) \simeq 2 \sqrt{\tau\left(1+\tau \rho^{2}\right)} \tilde{w}_{3}(\tau) .
\end{aligned}
$$

In the above, only $U^{L}$ and $\tilde{U}^{L}$ turn out to be affected by the terms linear in the struck nucleon's momentum $\eta$ through the expression $\lambda-\tau \rho$, whereas the transverse and axial functions are not. Higher-order terms in the $\eta$ expansion will modify $U^{T}, \tilde{U}^{T}$ and $\tilde{U}^{T^{\prime}}$ only as far as $\mathcal{D}^{T}$ and $\mathcal{D}^{T^{\prime}}$ are concerned, in contrast with the longitudinal case where they affect the whole of $U^{L}$ and $\tilde{U}^{L}$. In order to illustrate these items we show in Fig. 1 the electromagnetic and $\mathrm{PV}$ axial responses for both the $\mathrm{QE}$ and $N \rightarrow \Delta$ processes. The responses in the $\Delta$ region take into account the finite width of the resonance by the standard substitution

$$
R(q, \omega) \rightarrow \int_{W_{\min }}^{W_{\max }} \frac{1}{2 \pi} \frac{\Gamma(W)}{\left(W-m_{\Delta}\right)^{2}+\Gamma^{2}(W) / 4} R(q, \omega, W) d W
$$

where $R(q, \omega, W)$ is the response for a stable $\Delta$ of mass $W$; the invariant mass of the resonance $W$ ranges from $W_{\min }=m_{N}+m_{\pi}$ to $W_{\max }=\sqrt{\left(E_{F}+\omega\right)^{2}-\left(q-p_{F}\right)^{2}}$ and $\Gamma(W)$ is the usual P-wave $\Delta$ width [9]. We plot the exact RFG results given by (33, 34, 37) and those obtained from our expansion in $\eta$ of (39, 40, 43). In the case of the transversal and axial responses, the approximate result can hardly be distinguished from the exact one, showing that they are practically unaffected by the higher-order terms in $\eta$ (i.e., by $\left.\mathcal{D}^{T\left(T^{\prime}\right)}\right)$. The situation is different for the longitudinal response, as can be appreciated for the QE peak. Only here there is a contribution from linear terms, which improves the 
result of the leading-order expression (given by (39) without $2 \rho(\lambda-\tau \rho)$ ), but it is still insufficient to reproduce the exact result. On the other hand, both leading and next-toleading $N \rightarrow \Delta$ longitudinal responses vanish in the absence of $\mathcal{D}^{L}$. This is a consequence of having $G_{C, \Delta}(\tau)=0$, which implies $\left(1+\tau \rho^{2}\right) w_{2, \Delta}(\tau)-w_{1, \Delta}(\tau)=0$. Therefore, in the absence of the Coulomb form factor, the longitudinal $N \rightarrow \Delta$ response is proportional to $\mathcal{D}^{L}$. A non-vanishing $G_{C, \Delta}(\tau)$, even if small, avoids the cancellation and produces a contribution to $U^{L}$ in the $\Delta$ region that might be relevant at large momentum transfer [2].

Interestingly, the parts of $U^{L}$ and $\tilde{U}^{L}$ not proportional to $\mathcal{D}^{L}$ arise entirely from the longitudinal component of the nucleonic momentum

$$
\eta_{L} \simeq \frac{1}{\kappa}(\lambda-\tau \rho)
$$

On the other hand the transverse component of $\vec{\eta}$, namely

$$
\eta_{T}=\sqrt{\frac{\tau}{\kappa^{2}}(\epsilon+\lambda \rho)^{2}-1-\tau \rho^{2}},
$$

only contributes to $\mathcal{D}^{L}$. Actually, the longitudinal and transverse motion of a nucleon inside the RFG are not disconnected, but turn out to be linked by the kinematical constraint

$$
\eta_{L}=\lambda \sqrt{\frac{1}{\tau}\left(1+\tau \rho^{2}+\eta_{T}^{2}\right)}-\kappa \rho .
$$

Hence one cannot really associate each component of $\vec{\eta}$ to a particular contribution to the response. However, by removing the transverse nucleonic motion (setting $\eta_{T}=0$ ), the expressions (33) and (35) with $\mathcal{D}^{L}=0$ are exactly recovered.

Turning now to the the functions $\mathcal{D}^{L, T, T^{\prime}}$, we first notice that $\mathcal{D}^{L}$ and $\mathcal{D}^{T}$ arise from the second term on the right-hand side of (14), whereas $\mathcal{D}^{T^{\prime}}$ arises from the third one. By explicitly performing the calculation one arrives at the simple results

$$
\begin{aligned}
\mathcal{D}^{L}(\kappa, \tau)= & \mathcal{D}^{T}(\kappa, \tau)=\frac{1}{\epsilon_{F}-\epsilon_{0}} \int_{\epsilon_{0}}^{\epsilon_{F}} \eta_{T}^{2} d \epsilon \\
= & \frac{\tau}{\kappa^{2}}\left[(\lambda \rho+1)^{2}+(\lambda \rho+1)\left(1+\psi^{2}\right) \xi_{F}+\frac{1}{3}\left(1+\psi^{2}+\psi^{4}\right) \xi_{F}^{2}\right] \\
& -\left(1+\tau \rho^{2}\right)
\end{aligned}
$$

and

$$
\begin{aligned}
\mathcal{D}^{T^{\prime}}(\kappa, \tau) & =\frac{1}{\epsilon_{F}-\epsilon_{0}} \int_{\epsilon_{0}}^{\epsilon_{F}}\left(\sqrt{1+\frac{\eta_{T}^{2}}{1+\tau \rho^{2}}}-1\right) d \epsilon \\
& =\frac{1}{\kappa} \sqrt{\frac{\tau}{1+\tau \rho^{2}}}\left[1+\xi_{F}\left(1+\psi^{2}\right)+\lambda \rho\right]-1,
\end{aligned}
$$

which, when $\rho=1$, reduce to the corresponding quantities in the nucleonic sector. These results show that $\mathcal{D}^{L}$ and $\mathcal{D}^{T}$ just correspond to the mean square value of the transverse 
momentum of the nucleon, as already pointed out for the longitudinal channel [10], whereas $\mathcal{D}^{T^{\prime}}$ is related to the transverse kinetic energy of the nucleon when $\tau$ is small. Moreover, not only do the functions $\mathcal{D}^{L, T, T^{\prime}}$ start to get contributions from the quadratic terms of the $\eta$ expansion, but actually $\mathcal{D}^{L}$ and $\mathcal{D}^{T}$, unlike $\mathcal{D}^{T^{\prime}}$, are only contributed to by the quadratic terms. In order to further illustrate the different physical meaning of $\mathcal{D}^{L(T)}$ and $\mathcal{D}^{T^{\prime}}$, we go to the $\mathrm{QE}(\Delta)$ peak, where $\lambda=\tau \rho$. Then, from (48), (49), it follows that

$$
\mathcal{D}_{P}^{L(T)}(\tau)=\xi_{F}+\frac{1}{3\left(1+\tau \rho^{2}\right)} \xi_{F}^{2}
$$

and

$$
\mathcal{D}_{P}^{T^{\prime}}=\frac{\xi_{F}}{2\left(1+\tau \rho^{2}\right)}
$$

Since $\xi_{F}$ is quite small $\left(\xi_{F}=0.028\right.$ for $\left.{ }^{12} C\right)$, the second term of $(50)$ is negligible with respect to the first, so that $\mathcal{D}_{P}^{L(T)}$ is practically constant and independent of $\tau$ and $\rho$. On the other hand, $\mathcal{D}_{P}^{T^{\prime}}$ vanishes at large $\tau$, and is smaller at the $\Delta$ peak than at the $\mathrm{QE}$ one.

If the terms associated with $\mathcal{D}^{T}$ and $\mathcal{D}^{T^{\prime}}$ are neglected, then the PC transverse and the $\mathrm{PV}$ axial responses can be related for symmetric nuclei. For the QE process, one gets

$$
\frac{\tilde{R}^{T^{\prime}}}{R^{T}} \simeq \sqrt{\frac{\tau+1}{\tau}} \frac{G_{M, p}(\tau) \tilde{G}_{A, p}(\tau)+G_{M, n}(\tau) \tilde{G}_{A, n}(\tau)}{G_{M, p}^{2}(\tau)+G_{M, n}^{2}(\tau)},
$$

which can be simplified by neglecting the isoscalar form factors, leading to

$$
\frac{\tilde{R}^{T^{\prime}}}{R^{T}} \simeq \sqrt{\frac{\tau+1}{\tau}} \frac{G_{A}^{(1)}(\tau)}{G_{M}^{(1)}(\tau)}
$$

here $G_{M(A)}^{(1)}=G_{M(A), p}-G_{M(A), n}$ are the isovector magnetic and axial form factors. The relation (53) corresponds to the prescription given in formula (38) of [1], now proven to be valid up to next-to-leading order in a nucleon momentum expansion and at all orders in $\kappa$. Analogously, for the $N \rightarrow \Delta$ transition, we get

$$
\frac{\tilde{R}^{T^{\prime}}}{R^{T}} \simeq 4 \frac{\mu-1}{\mu+1} \frac{\sqrt{\tau\left(\tau \rho^{2}+1\right)}}{4 \tau+(1-\mu)^{2}} \frac{3 G_{E, \Delta}(\tau) \tilde{G}_{M, \Delta}^{A}(\tau)+G_{M, \Delta}(\tau) \tilde{G}_{E, \Delta}^{A}(\tau)}{G_{M, \Delta}^{2}(\tau)+3 G_{E, \Delta}^{2}(\tau)},
$$

which, under the assumption of $M 1$ dominance of the electromagnetic $N \rightarrow \Delta$ transition, becomes

$$
\frac{\tilde{R}^{T^{\prime}}}{R^{T}} \simeq 4 \frac{\mu-1}{\mu+1} \frac{\sqrt{\tau\left(\tau \rho^{2}+1\right)}}{4 \tau+(1-\mu)^{2}} \frac{\tilde{G}_{E, \Delta}^{A}(\tau)}{G_{M, \Delta}(\tau)} .
$$

Since $\mathcal{D}^{T}$ differs from $\mathcal{D}^{T^{\prime}}$ through terms of order $\eta_{F}^{4}$, the same will occur for the two responses; hence, the relationships (52)-(55) are valid to a remarkable accuracy in the RFG model. 
Now, as done above for $U$ and $\mathcal{D}$, it would appear natural also to explore the universal factor $R_{0}$ in the light of the $\eta$ expansion. However, in order to appreciate the importance of a fully relativistic treatment of the kinematics, we shall rather focus on the difference

$$
R_{0}-R_{0, n r}
$$

between the relativistic expression (34) and the non-relativistic one [12], which obtains by assuming non-relativistic energy-momentum relations and setting $1 /\left(\epsilon \epsilon^{\prime}\right) \approx 1$ in the energy denominators appearing in (7). With these approximations one gets

$$
R_{0, n r}=\frac{3 \mathcal{N}}{8 m_{N} \kappa \eta_{F}}\left(1-\psi_{n r}^{2}\right)
$$

with a non-relativistic scaling variable $\psi_{n r}$ whose square is given by [13]

$$
\psi_{n r}^{2}=\left(\frac{\eta_{0, n r}}{\eta_{F}}\right)^{2}
$$

in the Pauli unblocked region. In the above

$$
\eta_{0, n r}=\frac{2}{\mu-1}\left|\kappa-\sqrt{\mu\left[\kappa^{2}-(\mu-1) \lambda\right]}\right|
$$

represents the minimum momentum a struck nucleon should have to take part in the process. In passing it is easily checked that in the limit $\mu=1$ the non-relativistic scaling variable $\psi_{n r}=[\lambda / \kappa-\kappa] / \eta_{F}$ of 田 is recovered.

Next, we expand the function

$$
R_{0}-R_{0, n r}=\frac{3 \mathcal{N}}{4 m_{N} \kappa \eta_{F}^{3}}\left[\xi_{F}\left(1-\psi^{2}\right)-\frac{\eta_{F}^{2}}{2}\left(1-\psi_{n r}^{2}\right)\right]
$$

in powers of $\eta_{F}$. For this purpose we first notice that

$$
\kappa^{2}=\tau\left(1+\tau \rho^{2}\right)+2 \tau \rho(\lambda-\tau \rho)+\mathcal{O}\left(\eta_{F}^{2}\right) .
$$

Then, from (41) and (58), one gets that

$$
\xi_{F} \psi^{2}=\mathcal{O}\left(\eta_{F}^{2}\right),
$$

whereas

$$
\eta_{F}^{2} \psi_{n r}^{2}=4 \tau\left[\frac{\left.\sqrt{1+\tau \rho^{2}}-\sqrt{\mu\left[1+\tau \rho^{2}-\rho(\mu-1)\right.}\right]}{\mu-1}\right]^{2}+\mathcal{O}\left(\eta_{F}\right) .
$$

We thus reach the conclusion that the quantity

$$
R_{0}-R_{0, n r}=\frac{3 \mathcal{N}}{4 m_{N} \kappa \eta_{F}^{3}}\left\{2 \tau\left[\frac{\left.\sqrt{1+\tau \rho^{2}}-\sqrt{\mu\left[1+\tau \rho^{2}-\rho(\mu-1)\right.}\right]}{\mu-1}\right]^{2}+\mathcal{O}\left(\eta_{F}\right)\right\}
$$


not only does not vanish at leading order in $\eta_{F}$, but can be quite large indeed for high values of $\tau$. In particular, in the limit $\mu \rightarrow 1$, (64) yields

$$
R_{0}-R_{0, n r}=\frac{3 \mathcal{N}}{8 m_{N} \kappa \eta_{F}^{3}}\left[\frac{\tau^{3}}{1+\tau}+\mathcal{O}\left(\eta_{F}\right)\right]
$$

from which it follows that $R_{0, n r}$ should not be used if $\tau$ is not small.

We recall, however, that in the nucleonic sector an approximate scaling variable has been suggested in [13], namely

$$
\tilde{\psi}_{n r}=\frac{1}{\eta_{F}}\left[\frac{\lambda(1+\lambda)}{\kappa}-\kappa\right],
$$

which, although displaying the typical non-relativistic structure, actually incorporates some effects of relativity in the $\lambda(\lambda+1)$ term. The latter, when expanded in the nucleonic momentum, leads to

$$
\eta_{F} \tilde{\psi}_{n r}=\frac{\lambda-\tau}{\sqrt{\tau(1+\tau)}} .
$$

By inserting the above into (60) one obtains a difference of order $\eta_{F}^{2}$ between the exact and approximate $R_{0}$. This is another instance where using the leptonic variables $\kappa, \lambda$ and $\tau$ judiciously leads to improved convergence of expansions in $\eta_{F}$.

In summary, in this letter we have re-examined the interplay between the single-baryon and nuclear many-body content that takes place in electroweak interactions with nuclei. We have limited our focus to inclusive responses and have taken the RFG for guidance in attempting to gain insight into how these two elements enter. In particular, we have focused on expansions of the single-baryon cross sections in the one small parameter in the problem that is available in high-energy studies, namely, the dimensionless ratio $\eta_{F}=p_{F} / m_{N}$. Upon judiciously making use of the factor $\kappa^{2} / \tau=\left|q^{2} / Q^{2}\right|$, which is greater than unity and embodies some of the moving nucleon content in the problem, we are able to obtain singlebaryon cross sections whose leading terms contain no additional $\eta_{F}$-dependence beyond that implicit in factors such as $\kappa^{2} / \tau$, and have in next-to-leading order only contributions of order $\eta_{F}^{2}$, with no linear terms. Such corrections are usually so small that they can safely be neglected, providing considerable simplification to descriptions of inclusive electroweak processes. In contrast, for the nuclear many-body problem itself, as distinct from the single-baryon substructure, such non-relativistic expansions must be done with care.

\section{Acknowledgments}

This work is supported in part by the Bruno Rossi MIT-INFN exchange program, by the U.S. Department of Energy under Cooperative Research Agreement No. DF-FC0294ER40818 and by Spanish DGYCIT contract No. PB 96-0753. 


\section{References}

[1] T.W. Donnelly, M.J. Musolf, W.M. Alberico, M.B. Barbaro, A. De Pace and A. Molinari, Nucl. Phys. A 541 (1992) 525.

[2] J.E. Amaro, M.B. Barbaro, J.A. Caballero, T.W. Donnelly and A. Molinari, Nucl. Phys. A 657 (1999) 161.

[3] P. Amore, R. Cenni, T. W. Donnelly and A. Molinari, in preparation.

[4] W.M. Alberico, A. Molinari, T.W. Donnelly, E.L. Kronenberg and J.W. Van Orden, Phys. Rev. C 38 (1988) 1801.

[5] M.B. Barbaro, R. Cenni, A. De Pace, T.W. Donnelly and A. Molinari, Nucl. Phys. A 643 (1998) 137.

[6] T.W. Donnelly and I. Sick, Phys.Rev. C 60 (1999) 065502.

[7] J.E. Amaro, M.B. Barbaro, J.A. Caballero, T.W. Donnelly and A. Molinari, Nucl. Phys. A 643 (1998) 349.

[8] S. Jeschonnek and T.W. Donnelly, Phys. Rev. C 57 (1998) 2438.

[9] E. Oset, H. Toki and W. Weise, Phys. Rep. 83 (1982) 282.

[10] P. Amore, R. Cenni, T. W. Donnelly and A. Molinari, Nucl.Phys. A 615 (1997) 353.

[11] M.B. Barbaro, A. De Pace, T.W. Donnelly and A. Molinari, Nucl. Phys. A 569 (1994) 701.

[12] W.M. Alberico, R. Cenni and A. Molinari, La Rivista del Nuovo Cimento 1, n.4 (1978) .

[13] W.M. Alberico, T.W. Donnelly and A. Molinari, Nucl. Phys. A 512 (1990) 541. 


\section{Figures}
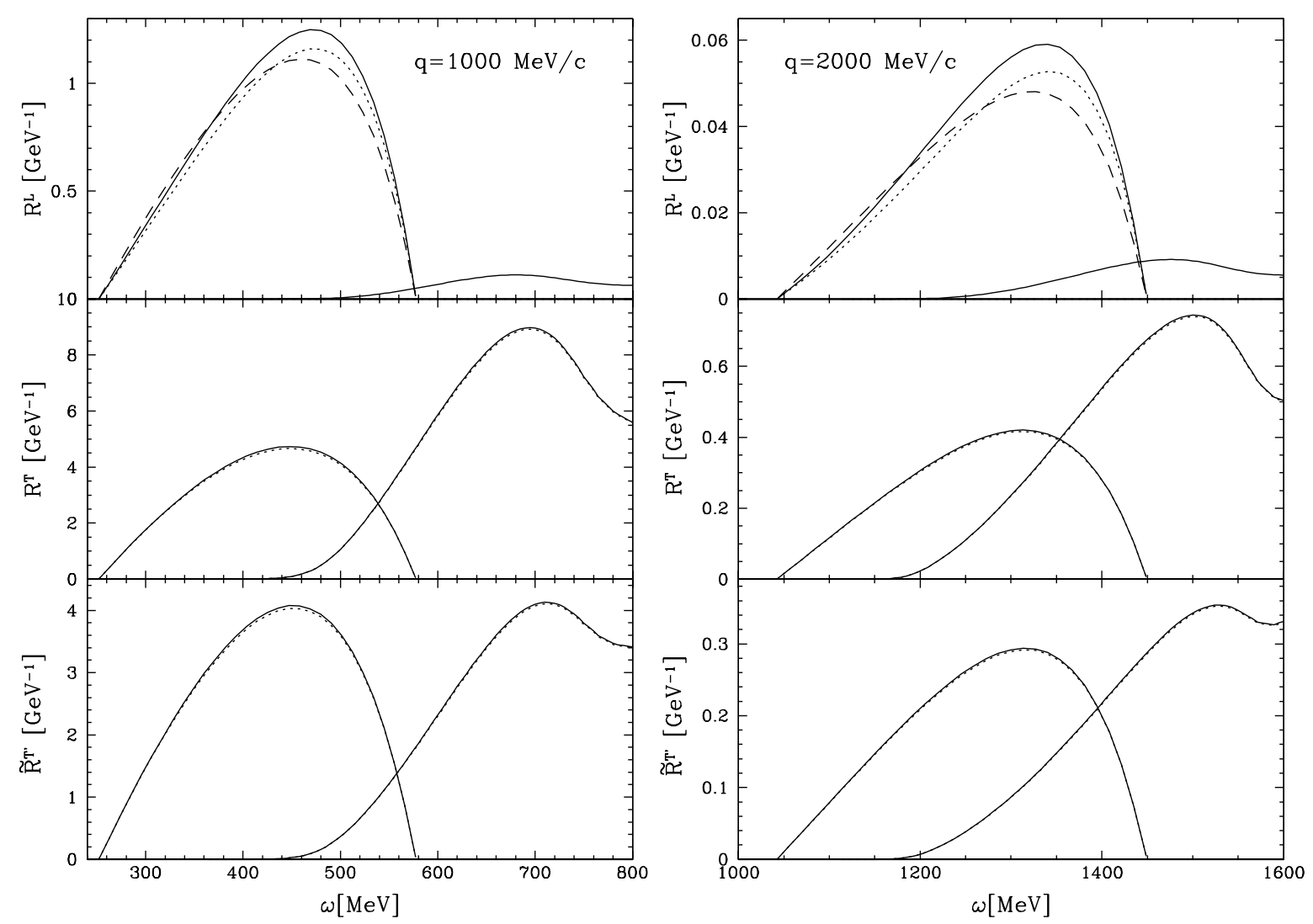

Figure 1: Electromagnetic and PV axial responses for ${ }^{12} \mathrm{C}\left(p_{F}=225 \mathrm{MeV} / \mathrm{c}\right)$. Every plot shows both the QE (left peak) and $\Delta$ (right peak) regions. The solid lines correspond to the exact responses from (33, 34, 37); the dotted lines show the result of the expansion in $\eta$ up to linear terms ((39, 40, 43) ); the dashed ones keep only the leading order in $\eta$ (which is different from the linear one only for the longitudinal response). 